\title{
Editorial
}

\section{Critical care audit}

Critical care utilization in Canada increased from 17 ICU patient days per thousand population in 1969 to 42 ICU patient days per thousand population in $1986 .{ }^{1}$ This represented approximately $8 \%$ of 1986 total inpatient costs. In the same year, intensive care utilization in the United States was 108 patient days per thousand population, accounting for $20 \%$ of all inpatient costs. This increase in utilization has taken place without knowledge of either the efficacy or cost-effectiveness of the care delivered. The need to control costs while demonstrating effective utilization of resources lends an urgency to the development of appropriate databases and, more importantly, processes through which outcome analysis can be translated into effective, high quality care at low cost. In their article in this issue, Byrick and Caskennette describe an automated system for critical care audit. The authors correctly identify two major elements of such utilization management and quality assurance systems. These are development and testing of an appropriate data base, and implementation of an audit process. The audit process must include definition of appropriate outcomes, interdisciplinary data analysis, development of an action plan to address important issues, and implementation of required changes. The final element in "closing the loop," namely evaluation of the impact of the audit process on quality of care and utilization is not addressed by the authors. In fact, there are few well-designed outcome studies which analyze the results of utilization review in the literature. ${ }^{2}$

Much of the information on utilization management comes from the United States where rapidly inflating health care costs and complex interrelationships of governmental and private payers have resulted in pressures to control costs while assuring quality care. Utilization management has evolved from early quality assurance efforts by providers, to increased third party involvement which has moved the process from retrospective, to concurrent, and even prospective (preadmission) review. Despite these massive efforts, the scientific merit of these endeavours remains unclear. A recent survey on utilization management ${ }^{3}$ revealed that ap-

From the Department of Surgery, Vancouver General Hospital, University of British Columbia
Judith A. Vestrup MD FRCSC

proximately half of Canadian acute care hospitals still rely on retrospective review only, and that most administrators were unclear about the impact of audit on either quality or rate of hospital admission. Despite the disappointment to date with audit processes, there is evidence in the critical care literature of progress in refinement of both outcome predictors and techniques for concurrent review.

Byrick and Caskennette have incorporated APACHE II, a prognostic scoring system widely used for outcome analysis, into their database. They have also included nursing workload indicators. They correctly identify one of the major challenges in evaluating critical care as being small patient volumes and heterogeneous patient populations. For example, in their series of 249 patients, 181 diagnoses were identified. The solution to this may lie in interinstitutional data sharing, using congruent data sets. Indeed, Wong and Knaus point to the capacity of APACHE II to control a case mix as one of its most valuable contributions. ${ }^{4} \mathrm{~A}$ similar approach can be found in the Major Trauma Outcome Study (MTOS) jointly sponsored by the American College of Surgeons and the Centres for Disease Control. ${ }^{5}$ This study included data on 80,544 trauma patients reported from 139 U.S. and Canadian hospitals. The data have already been used by participating hospitals to identify unexpected deaths and survivals for peer review. The MTOS has also been used by these hospitals to compare their standard of trauma care to that of other institutions. This model for development of normative standards may provide an essential key to further evaluative research in critical care.

While systems such as APACHE II permit outcome analysis on patient cohorts, there is now interest in developing patient-specific predictors. Ruttimann and Pollack, in collaboration with nine paediatric intensive care units, have developed and validated a "Pediatric Risk of Mortality Score" which provides a daily estimate of probability of patient survival over the next $24 \mathrm{hr}$. This technique may prove more valuable in supporting concurrent patient review. It had been shown that when provided with daily probability of survival data, critical care staff reduced the frequency of monitoring in a lowrisk paediatric ICU patient subset. This resulted in a reduced daily cost of care with no negative impact on outcome. ${ }^{7}$ A similar approach has been taken in identi- 
fying a low-risk otorhinolaryngology patient group in a surgical ICU. In this situation, low APACHE scores, little need for life-support systems, and minimal complications led to the recommendation that these patients need not be routinely admitted to the ICU. This approach of limiting access to expensive critical care resources for patient subsets for whom little or no benefit from such care and no impact on outcome is demonstrable may prove to be a very palatable way of controlling critical care costs.

Still another approach to utilization management which is gaining popularity in both the United States and Canada is the concept of case management. In this model, an interdisciplinary group of caregivers develops a critical path for a homogeneous patient population, including projected length of stay, timely and appropriate use of laboratory and monitoring services as well as discharge planning processes. ${ }^{9}$ Patients are then monitored on a daily basis and variances from the prescribed critical path identified and analysed. To date this approach has been most successful in populations with large case volumes, for example, isolated hip fracture in the elderly. However, this concept has now been applied to weaning from ventilation and tracheal extubation of a critical care population receiving mechanical ventilation. ${ }^{10}$ In this study, an interdisciplinary group regularly reviewed patients' progress toward weaning based on previously established care guidelines. The APACHE was used to control for case mix. Subsequent cost analysis showed this approach reduced ventilator days and ICU stay, while achieving considerable cost savings. There was no change in outcome as measured by mortality.

It is in this background of increased research activity directed toward evaluation of both the quality and resource implications of critical care delivery that Byrick and Caskennette developed their audit system. Their automated database was developed for a personal computer and required capture of a total of 388 data elements. Since data extraction and entry took place after hospital separation, they experienced a six- to ninemonth delay in report generation for audit purposes. This lack of timeliness presents a problem for retrospective, much less concurrent review. Management information systems currently available can provide for immediate download of data elements as well as linkage to other hospital-based computer systems. In particular, linkage of clinical data to a hospital's financial system will likely prove to be a very powerful tool in the future. Wong and Knaus $^{4}$ report that an automated APACHE III data management system will be available this year. With electronic linkages, physiological data can be downloaded directly from ICU monitors, thereby making individual patient information on APACHE score and risk prediction available on a daily basis. Such management systems will also address other problems identified by Byrick and Caskennette, such as the labour-intensive nature of their data acquisition. They also identify concerns with the accuracy and reproducibility of data coding which will be addressed, at least partially, with automated coding systems. Most importantly, the authors offer encouragement that the process of audit systems development can yield valuable information for utilization management. In addition, they report improved interdisciplinary communication and more effective unit management. As clinical activities are increasingly scrutinized, these insights into the process of audit system development as well as its potential limitations will prove invaluable to others as they move toward the goal of systematic evaluation and cost benefit analysis in critical care.

\section{Du bon usage des soins intensifs}

Au Canada, de 1969 à 1986, l'usage des lits de soins intensifs est passé de 17 jours-patients à 42 jours-patients par 1000 personnes-année, ${ }^{\prime}$ ce qui représente près de $8 \%$ des coûts d'hospitalisation. La même année, aux EtatsUnis, on utilisait les soins intensifs à raison de 108 jourspatients par 1000 personnes-année, y consacrant $20 \%$ des ressources d'hospitalisation. Cette augmentation de l'emploi des soins intensifs est survenue sans que l'on ait démontré leur efficacité. La nécessité de contenir les coûts en faisant preuve du bon usage des ressources exige qu'on développe au plus tôt les banques de données nécessaires à une analyse critique de nos résultats permettant de raffiner la qualité des soins sans y laisser sa chemise. Dans un article que vous lirez plus loin, Byrick et Caskennette nous présentent un système automatisé de révision des soins dits intensifs. Ils y soulignent deux éléments essentiels à tout système de gestion des ressources et d'assurance de la qualité, soit le développement d'une banque de données appropriées de même que la mise en place d'un processus de révision. Ce dernier doit comprendre la définition des résultats escomptés ou redoutés, l'analyse des données sur une base interdisciplinaire de même que l'établissement et la mise à l'épreuve d'une stratégie de correction des problèmes identifiés. Les auteurs n'abordent cependant pas l'élément nécessaire afin de fermer la boucle, soit 
l'évaluation de l'impact du processus de révision sur la qualité des soins et l'utilisation des ressources. Il faut dire que la recherche s'est encore peu penchée sur l'évaluation de ce dernier problème. ${ }^{2}$

Le corps des connaissances sur la gestion des ressources hospitalières nous vient des Etats-Unis où l'ascension des coûts des soins de santé couplée à des relations complexes entre payeurs publics et privés a entraîné de fortes pressions en vue de contenir la facture tout en assurant des soins de qualité. Avec la participation grandissante des tiers-payeurs, le processus d'assurance de la qualité initié à l'origine par le personnel soignant s'est transformé en un exercice de gestion des ressources dont la nature rétrospective s'est progressivement enrichie d'un volet contemporain et même prospectif. Une revue récente sur la gestion des ressources ${ }^{3}$ nous révèle que près de la moitié des hôpitaux canadiens de soins aigus ne font qu'une étude rétrospective de leur utilisation et que la majorité des administrateurs ne peuvent préciser l'impact de cet exercice sur la qualité des soins et le taux d'hospitalisation. Toutefois, même si en général jusqu'à ce jour les processus de révision peuvent avoir déçu, il semble que dans le monde des soins intensifs, à tout le moins, apparaissent de meilleures techniques de révision simultanée aux soins et même certains marqueurs prospectifs fiables.

Dans leur étude, Byrick et Caskennette ont pris en compte le score APACHE II, un indicateur pronostique largement employé dans l'analyse des résultats et ils ont aussi employé un indice de la lourdeur des cas en fonction de la demande en soins infirmiers. Ils ont souligné avec justesse une des difficultés de l'évaluation des soins intensifs, soit le petit nombre de patients et la diversité des cas. Ainsi leurs 249 patients arboraient 181 diagnostics différents. Le regroupement des données entre différents centres de soins pourrait peut-être pallier à cette difficulté, le score APACHE II pouvant alors servir de base de comparaison ainsi que l'ont démontré Wong et Knaus. ${ }^{4}$ C'est sur ce principe qu'était basée la « Major Trauma Outcome Study " (MTOS) mise de l'avant par l'American College of Surgeons et le Centre for Disease Control. ${ }^{5}$ On peut y retrouver des données sur 80544 cas de traumatismes en provenance de 139 hôpitaux canadiens et américains. Les hôpitaux participants ont déjà pu se servir des données ainsi colligées afin d'identifier les cas de décès et de survie particulièrement saisissants dans le but d'en faire l'analyse détaillée. Ils ont aussi pu comparer leurs standards de traitement à ceux des autres institutions. Ce modèle pourra d'ailleurs servir à l'établissement de standards normatifs, étape probablement essentielle aux efforts de recherche sur l'évaluation des soins intensifs.

Alors que le système APACHE II contribue à l'étude des résultats obtenus sur des cohortes de patients, certains tiennent à développer des indicateurs de risque applicables à des individus. Ainsi, Ruttimann et Pollack ont crée et validé dans neuf unités de soins intensifs pédiatriques un "Pediatric Risk of Mortality Score » qui évalue quotidiennement le risque de décès des 24 prochaines heures. ${ }^{6}$ Voilà un bel exemple d'analyse de gestion des ressources simultanée à l'épisode de soins puisque, muni quotidiennement de l'indicateur de risque de décès, le personnel soignant diminuaient la lourdeur du monitorage chez les enfants les moins à risque. Cela permit de réduire la facture quotidienne sans encourir de conséquence fâcheuse. ${ }^{7}$ On suivit une démarche semblable en identifiant le faible risque que représentait une certaine population de patients ayant subi une intervention otorhino-laryngologique admis à l'unité de soins intensifs chirurgicaux. Avec leur score APACHE modeste, un taux de complication marginal et leur faible utilisation d'équipement de survie lourd, on put mettre fin à l'admission routinière de ces patients aux soins intensifs. Dans une optique de contrôle des coûts, on devrait limiter l'admission aux soins intensifs aux patients faisant partie de groupes à risque chez qui on a démontré les avantages de cette démarche.

Le plan de soins est une autre forme de gestion des ressources qui gagne des adeptes tant aux Etats-Unis qu'au Canada. Une équipe multidisciplinaire détermine un plan approprié à une certaine catégorie de patients et y prévoit la durée de séjour, l'usage des examens de laboratoire et de monitorage, de même que la procédure de conge. ${ }^{9}$ On suit alors chaque patient quotidiennement et on identifie tout écart du plan de soins afin de l'analyser. Jusqu'à maintenant, on a pu démontrer l'intérêt de cette approche pour les pathologies fréquentes telles les fractures isolées de la hanche chez les personnes âgées. On s'en est aussi servi pour le sevrage du respirateur aux soins intensifs. ${ }^{10}$ Dans cette demière étude, une équipe multidisciplinaire révisait régulièrement les progrès de chaque patient en fonction d'un protocole de soins établi d'avance. On s'y servait du score APACHE pour contrôler certaines variables. L'analyse des résultats a permis d'identifier une diminution de la période de ventilation mécanique et de séjour aux soins intensifs ainsi que des économies substantielles. Les mortalité des patients n'en était pas affectée.

C'est dans ce contexte d'une plus grande sensibilité quant à l'évaluation de la qualité et des coûts des soins intensifs que Byrick et Caskennette ont développé leur système de révision. C'est à partir d'un micro-ordinateur qu'ils généraient une banque de donnée formée de 388 éléments par patient. Comme ils y entraient leurs données après le congé des patients, il encoururent des délais de six à neuf mois avant de pouvoir produire un rapport. 
Ces délais retardent le processus de révision toutefois il existe maintenant des systèmes de gestion de l'information qui permettent la communication immédiate de certaines données vers les ordinateurs centraux d'un hôpital dont l'accès est devenu facile. D'ailleurs le couplage de données d'ordre clinique au système de gestion financière de l'hôpital en fera d'ici peu un outil apprécié. Wong et Knaus ${ }^{4}$ annoncent pour bientôt l'arrivée du logiciel APACHE III qui s'alimentera directement à partir des moniteurs de soins intensifs permettant la mise à jour quotidienne du score APACHE de chaque patient et en prédisant le risque associé. Ce type de système automatisé permettra de réduire la somme de travail clérical nécessaire à l'entrée des données et minimisera le risque d'erreur d'encodage.

Byrick et Caskennette nous confirment que leur processus de révision génère de l'information utile à la gestion des ressources. En prime, ils ont constaté une amélioration de la communication entre disciplines et une meilleure gestion de l'unité de soins. Les gestionnaires n'ont pas fini de vouloir passer à la loupe nos activités cliniques et ces connaissances nouvelles qu'on peut développer sur les avantages et limites des processus de révision nous permettent d'avancer vers l'évaluation systématique des coûts et bénéfices des soins intensifs.

\section{References}

1 Jacobs, P, Noseworthy TW. National estimates of intensive care utilization and costs: Canada and the United States. Crit Care Med 1990; 18: 1282-6.

2 Ermann D. Hospital utilization review: past experience, future directions. Journal of Health Politics, Policy and Law 1988; 13: 683-704.

3 Anderson $G$, Sheps $S B$, Cardiff $K$. Hospital-based utilization management: a cross-Canada survey. Can Med Assoc J 1990; 143: 1025-30.

4 Wong DT, Knaus WA. Predicting outcome in critical care: the current status of the APACHE prognostic scoring system. Can J Anaesth 1991; 38: 374-83.

5 Champion HR, Copes WS, Sacco WJ, et al. The major trauma outcome study: establishing national norms for trauma care. J Trauma 1990; 30: 1356-65.

6 Ruttimann UE, Pollack MM. Objective assessment of changing mortality risks in pediatric intensive care unit patients. Crit Care Med 1991; 19: 474-83.

7 Pollack MM, Getson PR. Pediatric critical care cost containment: combined actuarial and clinical program. Crit Care Med 1991; 19: 12-20.

8 Muakkassa FF, Fakhry SM, Rutledge R, Hsu H, Meyer $A A$. Cost-effective use of microcomputers for quality assurance and resource utilization in the surgical intensive care unit. Crit Care Med 1990; 18: 1243-7.
9 Zander $K$. Nursing case management: strategic management of cost and quality outcomes. Journal of Nursing Administration 1988; 18: 23-30.

10 Cohen IL, Bari N, Strosberg MA, et al. Reduction of duration and cost of mechanical ventilation in an intensive care unit by use of a ventilatory management team. Crit Care Med 1991; 19: 1278-84. 